$2-1-2012$

\title{
Free Torus Actions and Two-Stage Spaces
}

\author{
Barry Jessup \\ University of Ottawa \\ Gregory Lupton \\ Cleveland State University, g.lupton@csuohio.edu
}

Follow this and additional works at: https://engagedscholarship.csuohio.edu/scimath_facpub

Part of the Mathematics Commons

How does access to this work benefit you? Let us know!

\section{Publisher's Statement}

(C2004 Cambridge Philosophical Society. https://www.cambridge.org/core/journals/

mathematical-proceedings-of-the-cambridge-philosophical-society/article/free-torus-actionsand-twostage-spaces/B5C15F497D81563FE42B1CD75CDBA52E

\section{Repository Citation}

Jessup, Barry and Lupton, Gregory, "Free Torus Actions and Two-Stage Spaces" (2012). Mathematics Faculty Publications. 191.

https://engagedscholarship.csuohio.edu/scimath_facpub/191

This Article is brought to you for free and open access by the Mathematics and Statistics Department at EngagedScholarship@CSU. It has been accepted for inclusion in Mathematics Faculty Publications by an authorized administrator of EngagedScholarship@CSU. For more information, please contact library.es@csuohio.edu. 


\title{
Free torus actions and two-stage spaces
}

\author{
BY BARRY JESSUP \\ AND GREGORY LUPTON†
}

\begin{abstract}
We prove the toral rank conjecture of Halperin in some new cases. Our results apply to certain elliptic spaces that have a two-stage Sullivan minimal model, and are obtained by combining new lower bounds for the dimension of the cohomology and new upper bounds for the toral rank. The paper concludes with examples and suggestions for future work.
\end{abstract}

\section{Introduction}

A well-known conjecture due to Halperin concerns torus actions on a space (see [Halb, problem 1.4]). If $X$ is a space on which an $n$-dimensional torus acts, we say the action is almost-free if each isotropy subgroup is a finite group. The largest integer $n \geqslant 1$ for which $X$ admits an almost-free $n$-torus action is called the toral rank of $X$ and is denoted rk $(X)$. If $X$ does not admit an almost-free circle action, then $\operatorname{rk}(X)=0$. Halperin's conjecture gives an upper bound for the toral rank of $X$ in terms of its cohomology, as follows:

Conjecture 1·1 (The toral rank conjecture). If $X$ is simply connected, then

$$
\operatorname{dim} H(X ; \mathbb{Q}) \geqslant 2^{\mathrm{rk}(X)} .
$$

We shall henceforth assume that all our spaces are 1-connected, finite cell complexes. There are some technical conditions on the topology of the space $X$ in Halperin's original formulation, but as these are satisfied by finite cell complexes, we will not mention them explicitly here.

The main tool we shall use is the Sullivan minimal model, and a basic reference is $[$ FHT]. For our purposes, we note that to any 1 -connected space $X$ there corresponds, in a contravariant way, a commutative differential graded algebra (unique up to isomorphism) $(\Lambda W, d)$, called the minimal model of $X$, which algebraically models the rational homotopy type of the space. By $\Lambda W$ we mean the free graded commutative 
algebra generated by the graded vector space $W$. The differential $d$ of any element of $W$ is a polynomial in $\Lambda W$ with no linear term, which in particular means that there is a homogeneous basis $\left\{w_{i}\right\}_{i \geqslant 1}$ of $W$ for which $d w_{i} \in \Lambda W_{<i}$, where $W_{<i}$ denotes the subspace of $W$ generated by $\left\{w_{j}\right\}_{j<i}$. (We will also occasionally consider models $(\Lambda W, d)$ which satisfy this latter nilpotence condition but where the polynomial $d w$ may have a linear term.) This contravariant correspondence yields an equivalence between the homotopy category of 1-connected rational spaces of finite type and that of 1-connected rational commutative differential graded algebras of finite type. In particular, if $(\Lambda W, d)$ is the minimal model of $X$, then $H(\Lambda W, d) \cong H(X ; \mathbb{Q})$ as graded algebras, and $W \cong \pi(X) \otimes \mathbb{Q}$ as graded vector spaces. Moreover, we remark that a series of results of Halperin and others implies that if $H(\Lambda W, d)$ and $W$ are finitedimensional, then $H(\Lambda W, d)$ must satisfy Poincaré duality, and $(\Lambda W, d)$ is the minimal model of some closed smooth manifold. In all cases of interest to us here, the space of generators $W$ will be finite-dimensional. If $\left\{w_{1}, \ldots, w_{m}\right\}$ is a homogeneous basis for $W$, then we will write $W=\left\langle w_{1}, \ldots, w_{m}\right\rangle$, and we also denote $\Lambda W$ by $\Lambda\left(w_{1}, \ldots, w_{m}\right)$ in this case, often omitting explicit reference to the differential. We note that models with $W$ and $H(\Lambda W, d)$ both finite-dimensional are called elliptic, and a space $X$ with an elliptic minimal model is called an elliptic space. Topologically, this means that both $\pi(X) \otimes \mathbb{Q}$ and $H(X ; \mathbb{Q})$ are finite dimensional.

Conjecture $1 \cdot 1$ is already known to hold for homogeneous spaces $G / H$, for $G$ connected, and $H$ closed and connected [Halb, proposition 1.5]. Such spaces have two-stage minimal models [FHT, proposition $15 \cdot 16]$, where a minimal model $(\Lambda W, d)$ is said to be two-stage if $W$ decomposes as $W \cong U \oplus V$ with $d U=0$ and $d V \subseteq \Lambda U$. By the remark in the previous paragraph, it is easy to see that there are many other examples of spaces, indeed smooth manifolds, with two-stage models, and it is these spaces that we shall study (see for example Corollary $2 \cdot 4$ ).

We note for later reference that there may be several ways to display a minimal model as a two-stage model. In particular, a generator in $V$ that is a cocycle could just as well be included in $U$. We will generally be interested in choosing a two-stage decomposition in which $V$ is as large as possible.

By a K-S extension (Koszul-Sullivan), or simply an extension, we mean a sequence of the form

$$
\left(\Lambda W_{1}, d_{1}\right) \longrightarrow\left(\Lambda W_{1} \otimes \Lambda W_{2}, D\right) \longrightarrow\left(\Lambda W_{2}, d_{2}\right)
$$

with $\left(\Lambda W_{1}, d_{1}\right)$ a minimal model, in which $D$ restricts to $d_{1}$ on $\Lambda W_{1} \otimes 1$, and for which there is an ordered basis of $W_{2}=\left\langle w_{1}, w_{2}, \ldots\right\rangle$ with $D w_{i} \in \Lambda W_{1} \otimes \Lambda\left(w_{1}, \ldots, w_{i-1}\right)$ for each $i$. A $\mathrm{K}-\mathrm{S}$ extension is the minimal-model analogue of a Serre fibration (cf. [FHT, section 15(a)]), and for this reason, $\Lambda W_{1}, \Lambda W_{1} \otimes \Lambda W_{2}$ and $\Lambda W_{2}$ are known respectively as the base, the total space and the fibre of the extension.

The connection between minimal models and the toral rank is originally due to Allday and Halperin [AH] (cf. [Hal, proposition 4.2]): If $X$ has minimal model $(\Lambda W, d)$ and admits an $n$-dimensional torus action, then there is an extension

$$
\Lambda A_{n} \longrightarrow \Lambda A_{n} \otimes \Lambda W \longrightarrow \Lambda W
$$

in which $A_{n}=\left\langle a_{1}, \ldots, a_{n}\right\rangle$ with each $a_{i}$ of degree 2 . If the action is almost free, then $\operatorname{dim} H\left(\Lambda A_{n} \otimes \Lambda W, D\right)$ is finite-dimensional [Hal, proposition $4 \cdot 2$ ]. In principle, this result allows an upper bound for $\operatorname{rk}(X)$ to be obtained by a direct analysis of the 
minimal model of $X$, and this direct approach has been carried out to great effect in some situations. In general, however, the computational problems involved here appear to be quite substantial.

However, since we are really only considering the rational homotopy type of $X$, we are led to the following variation of the toral rank: the rational toral rank $\operatorname{rk}_{0}(X)$ of $X$ is defined by $\operatorname{rk}_{0}(X)=\max \left\{\operatorname{rk}(Y) \mid Y \simeq_{\mathbb{Q}} X\right\}$. Clearly, we have $\operatorname{rk}_{0}(X) \geqslant \operatorname{rk}(X)$, hence an upper bound on $\operatorname{rk}_{0}(X)$ will serve as one on $\mathrm{rk}(X)$. See [Halb, proposition $4 \cdot 2$ ] for the precise relationship between these two numbers. The characterization of $\mathrm{rk}_{0}(X)$ in terms of a minimal model of $X$ is as follows. If $(\Lambda W, d)$ is a minimal model of $X$, then $\operatorname{rk}_{0}(X)$ is the largest $n$ (if such exists) for which there is a K-S-extension of the form (1), for which $\operatorname{dim} H\left(\Lambda A_{n} \otimes \Lambda W, D\right)$ is finite-dimensional. We will also denote $\operatorname{rk}_{0}(X)$ by $\operatorname{rk}_{0}(\Lambda W)$.

Now we briefly summarize the contents of the paper. In Section 2 , Theorem $2 \cdot 8$ gives a lower bound for the dimension of the cohomology of any space with an elliptic two-stage minimal model. This result is proved using standard tools familiar from algebraic topology, namely the Wang sequence and the Serre spectral sequence. Also in Section 2 we give two results that, under certain additional hypotheses, give upper bounds on the rational toral rank-hence on the toral rank-of a space with two-stage minimal model (Theorem 2.9 and Theorem 2.10). Combining these results allows us to establish Conjecture $1 \cdot 1$ in some new cases (Corollary 2.11). In addition, our results can be used to provide new and essentially elementary proofs of the conjecture in some previously-known cases, which we discuss after Theorem $2 \cdot 3$. In Section 3, we give a number of examples and suggest several directions for future investigation.

\section{Cohomology and toral rank of two-stage minimal models}

In this section we give several results about the cohomology and the toral rank of two-stage minimal models.

\subsection{Cohomology of two-stage minimal models}

We begin by establishing a lower bound for the dimension of the cohomology of an elliptic space with two-stage minimal model. Our bound is of a form similar to that featured in the toral rank conjecture.

If $\Lambda(U, V)$ is an elliptic two-stage minimal model, then the Mapping theorem [FHT, p. 375] implies that $V$ has generators of odd degree only. On the other hand, $U$ may have generators of odd or even degree. For the remainder of the paper, unless otherwise specified, we assume that all minimal models are two-stage, elliptic. For details about elliptic spaces and their minimal models, see [FHT, section 32].

First, we establish our lower bound in a special case. The main result is then established by reducing to this special case.

Proposition 2.1. Let $\Lambda(U, V)$ be a two-stage, elliptic minimal model with odd degree generators only and suppose that $d: V \rightarrow \Lambda^{2} U$ is an isomorphism. Then $\operatorname{dim} H(\Lambda(U, V)) \geqslant 2^{\operatorname{dim} V}$.

Proof. Suppose that $\operatorname{dim} U=n$. Since $d: V \rightarrow \Lambda^{2} U$ an isomorphism, and since $\Lambda U$ is an exterior algebra, we have $\operatorname{dim} V=\left(\begin{array}{c}n \\ 2\end{array}\right)$. Thus we must show that $\operatorname{dim} H(\Lambda(U, V)) \geqslant$ 


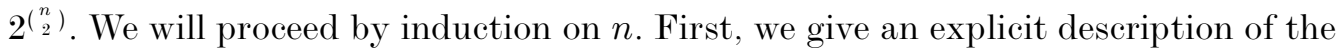
model $\Lambda(U, V)$.

Write $\Lambda(U, V)$ as $\mathcal{M}_{n}=\left(\Lambda\left(U_{n}, V_{n}\right), d_{n}\right)$, with $U_{n}=\left\langle u_{1}, \ldots, u_{n}\right\rangle$ and $V_{n}=$ $\left\langle\left\{v_{i, j}\right\}_{1 \leqslant i<j \leqslant n}\right\rangle$. The differential is $d_{n}\left(U_{n}\right)=0$ and $d_{n}\left(v_{i, j}\right)=u_{i} u_{j}$ for each $0 \leqslant i<$ $j \leqslant n$. For $n=1$, we have $\operatorname{dim} U_{1}=1$ and $V_{1}=0$, and the proposition is trivial. For $n=2$, we have $\mathcal{M}_{2}=\Lambda\left(u_{1}, u_{2}, v_{1,2}\right)$. It is easily checked that $\operatorname{dim} H\left(\mathcal{M}_{2}\right)=6 \geqslant 2^{1}$. This starts the induction.

Now suppose inductively that $\operatorname{dim} H\left(\mathcal{M}_{n}\right) \geqslant 2^{\left(\begin{array}{c}n \\ 2\end{array}\right)}$ for some $n \geqslant 2$. We adjust the notation somewhat and write $\mathcal{M}_{n+1}$ as follows. Write $U_{n+1}=\left\langle u_{0}, u_{1}, \ldots, u_{n}\right\rangle=$ $\left\langle u_{0}\right\rangle \oplus U_{n}$. Also, set $V_{0}=\left\langle v_{0,1}, \ldots, v_{0, n}\right\rangle$, so that $V_{n+1}=V_{0} \oplus V_{n}$. The differential $d_{n+1}$ of $\mathcal{M}_{n+1}$ extends that of $\mathcal{M}_{n}$. Thus, we have $d_{n+1}\left(U_{n+1}\right)=0, d_{n+1}\left(v_{i, j}\right)=d_{n}\left(v_{i, j}\right)=u_{i} u_{j}$ for $1 \leqslant i<j \leqslant n$ and $d_{n+1}\left(v_{0, j}\right)=u_{0} u_{j}$ for $1 \leqslant j \leqslant n$.

Further, if $\overline{\mathcal{M}}_{n+1}$ denotes $\mathcal{M}_{n} \otimes \Lambda V_{0}$, we have the following extension:

$$
\Lambda u_{0} \longrightarrow\left(\mathcal{M}_{n+1}, d_{n+1}\right) \longrightarrow\left(\overline{\mathcal{M}}_{n+1}, \bar{d}_{n+1}\right) .
$$

Consider the short exact sequence of differential graded vector spaces

$$
0 \longrightarrow \overline{\mathcal{M}}_{n+1} \stackrel{j}{\longrightarrow} \mathcal{M}_{n+1} \stackrel{p}{\longrightarrow} \overline{\mathcal{M}}_{n+1} \longrightarrow 0,
$$

where $p$ denotes the projection and $j$ the map defined by $j(\chi)=u_{0} \chi$ for $\chi \in \Lambda W$. The ensuing long exact sequence in cohomology has a connecting homomorphism that may be described as follows: on $\overline{\mathcal{M}}_{n+1}$, define $\theta^{*}: \overline{\mathcal{M}}_{n+1} \rightarrow \overline{\mathcal{M}}_{n+1}$ by $u_{0} \theta=d_{n+1}-\bar{d}_{n+1}$. Then, one can easily check that $\theta$ is actually a derivation of the algebra $\overline{\mathcal{M}}_{n+1}$ of degree $1-\left|u_{0}\right|$ which commutes with $\bar{d}_{n+1}$, and so induces a map $\theta^{*}: H\left(\overline{\mathcal{M}}_{n+1}\right) \rightarrow$ $H^{*-\left|u_{0}\right|+1}\left(\overline{\mathcal{M}}_{n+1}\right)$ which is the connecting homomorphism of (3). We will call the derivation $\theta^{*}$ the Wang derivation for (2), for reasons indicated in Remark $2 \cdot 2$ below.

This Wang derivation can be explicitly computed: because $d_{n+1}\left(V_{0}\right) \subseteq u_{0} \cdot U_{n}$, we see that $H\left(\overline{\mathcal{M}}_{n+1}, \bar{d}_{n+1}\right)=H\left(\mathcal{M}_{n}\right) \otimes \Lambda V_{0}$. Now, for $\chi \in \mathcal{M}_{n} \otimes \Lambda V_{0}$, we find that $\theta\left(\mathcal{M}_{n}\right)=0$, directly from the definition. Since $d_{n+1}\left(v_{0, i}\right)=u_{0} u_{i}$, we have $\theta\left(v_{0, i}\right)=u_{i}$ for each $i=1, \ldots, n$. On passing to cohomology, therefore, we find that $\theta^{*}\left(H\left(\mathcal{M}_{n}\right)\right)$ $=0$ and $\theta^{*}\left(v_{0, i}\right)=u_{i} \in H\left(\mathcal{M}_{n}\right)$ for $i=1, \ldots, n$.

As usual, we can condense the long exact sequence from (3) into

$$
0 \longrightarrow \operatorname{coker} \theta^{*} \longrightarrow H\left(\mathcal{M}_{n+1}\right) \longrightarrow \operatorname{ker} \theta^{*} \longrightarrow 0,
$$

so that to complete the induction, it simply remains to show that

$$
\operatorname{dim} \operatorname{ker} \theta^{*}+\operatorname{dim} \operatorname{coker} \theta^{*}=2 \operatorname{dim} \operatorname{ker} \theta^{*}
$$

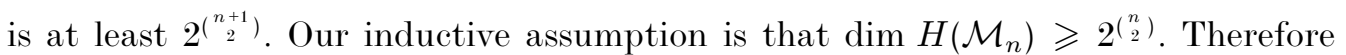
$\operatorname{dim}\left(H\left(\mathcal{M}_{n}\right) \cdot \Lambda V_{0}\right) \geqslant 2^{\left(\begin{array}{c}n \\ 2\end{array}\right)} \times 2^{n}$. Since $\left(\begin{array}{c}n \\ 2\end{array}\right)+n=\left(\begin{array}{c}n+1 \\ 2\end{array}\right)$, our inductive assumption

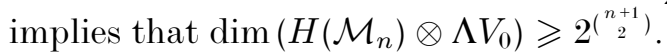

We claim that $\left(\theta^{*}\right)^{2}=0$, after which elementary linear algebra completes the proof. The key observation here is the following: denote by $\left[u_{i}\right] \in H\left(\mathcal{M}_{n}\right)$ the class represented by the cocycle $u_{i}$. Then the ideal of $H\left(\mathcal{M}_{n}\right)$ generated by all two-fold products $\left\{\left[u_{i}\right]\left[u_{j}\right]\right\}_{1 \leqslant i<j \leqslant n}$ is zero. This follows since each product $u_{i} u_{j}$ is a boundary, namely $d_{n}\left(v_{i, j}\right)$. Recall that $\theta^{*}\left(H\left(\mathcal{M}_{n}\right)\right)=0$, whilst $\theta^{*}\left(V_{0}\right)=U_{n} \subseteq H\left(\mathcal{M}_{n}\right)$. Thus, $\left(\theta^{*}\right)^{2}\left(H\left(\mathcal{M}_{n}\right) \otimes \Lambda V_{0}\right)$ is contained in the ideal generated by the two-fold products $\left\{\left[u_{i}\right]\left[u_{j}\right]\right\}_{1 \leqslant i<j \leqslant n}$, which we observed is zero. We conclude that $\left(\theta^{*}\right)^{2}=0$. 
But $\left(\theta^{*}\right)^{2}=0$ implies $2 \operatorname{dim} \operatorname{ker} \theta^{*} \geqslant \operatorname{dim}\left(H\left(\mathcal{M}_{n}\right) \otimes \Lambda V_{0}\right)$. Therefore, we have

$$
\operatorname{dim} H\left(\mathcal{M}_{n+1}\right)=2 \operatorname{dim} \operatorname{ker} \theta^{*} \geqslant \operatorname{dim}\left(H\left(\mathcal{M}_{n}\right) \otimes \Lambda V_{0}\right) \geqslant 2_{2}^{\left(\begin{array}{c}
n+1 \\
2
\end{array}\right)} .
$$

This completes the induction.

Remark $2 \cdot 2$. Topologically, the extension sequence (2) corresponds to a fibration with base an odd sphere $S^{2 r+1}$, and the long exact sequence in cohomology induced from (3) corresponds to the Wang sequence of the fibration, in the usual sense of a fibration with base a sphere. The derivation $\theta^{*}$ corresponds to the Wang derivation of the fibration, again in the usual sense (cf. [Whi, p. 319]).

As promised, the main result is now obtained by reducing the general two-stage case to that of Proposition $2 \cdot 1$.

Theorem $2 \cdot 3$. Suppose $\Lambda(U, V)$ is any two-stage, elliptic minimal model. Then

$$
\operatorname{dim} H(\Lambda(U, V)) \geqslant 2^{\operatorname{dim} V-\operatorname{dim} U^{\text {even }}} .
$$

Proof. Given $\Lambda(U, V)$, form the following extension sequence:

$$
\Lambda(U, V) \longrightarrow \Lambda(U, V) \otimes \Lambda(\bar{W}, W), D \longrightarrow \Lambda(\bar{W}, W), \bar{D}=0
$$

in which $D: \bar{W} \rightarrow U^{\text {even }}$ and $d: W \rightarrow \Lambda^{2}\left(U^{\text {odd }}\right)$ are vector space isomorphisms. This extension sequence has an associated Serre spectral sequence, obtained by filtering the total space by degree in $\Lambda(U, V)$. This spectral sequence has $E_{2}$-term isomorphic to $H(\Lambda(U, V), d) \otimes H(\Lambda(\bar{W}, W), \bar{D}=0) \cong H(\Lambda(U, V), d) \otimes \Lambda(\bar{W}, W)$ and it converges to $H(\Lambda(U, V) \otimes \Lambda(\bar{W}, W), D)$ (cf. [FHT, section 18], especially example 2 and exercise 2 ). Since the $E_{2}$-term of this spectral sequence must be at least as large as the term that it converges to, we have the following inequality:

$$
\operatorname{dim}(H(\Lambda(U, V), d)) \cdot 2^{\operatorname{dim} \bar{W}} \cdot 2^{\operatorname{dim} W} \geqslant \operatorname{dim}(H(\Lambda(U, V) \otimes \Lambda(\bar{W}, W), D)) .
$$

We now claim that $(\Lambda(U, V) \otimes \Lambda(\bar{W}, W), D)$ and $\left(\Lambda\left(U^{\text {odd }}, W\right) \otimes \Lambda(V), D \otimes 1\right)$ are quasi-isomorphic. To see this, argue as follows. First, display the middle term of (4) as the middle term of the following extension sequence:

$$
\left(\Lambda\left(U^{\text {even }}, \bar{W}\right), D\right) \longrightarrow(\Lambda(U, V) \otimes \Lambda(\bar{W}, W), D) \longrightarrow\left(\Lambda\left(U^{\text {odd }}, W, V\right), \bar{D}\right),
$$

in which $\left(\Lambda\left(U^{\text {even }}, \bar{W}\right), D\right)$ is an acyclic model. It follows that the projection $(\Lambda(U, V) \otimes \Lambda(\bar{W}, W), D) \rightarrow\left(\Lambda\left(U^{\text {odd }}, W, V\right), \bar{D}\right)$ is a quasi-isomorphism. Next, define a map of models $\phi:\left(\Lambda\left(U^{\text {odd }}, W\right) \otimes \Lambda(V), D \otimes 1\right) \rightarrow\left(\Lambda\left(U^{\text {odd }}, W, V\right), \bar{D}\right)$ as follows. Set $\phi$ to be the identity on $\Lambda\left(U^{\text {odd }}, W\right)$. For each generator $v_{i} \in V$, we have $\bar{D}\left(v_{i}\right) \in \Lambda^{\geqslant 2} U^{\text {odd }} \subseteq \bar{D}(W) \cdot \Lambda\left(U^{\text {odd }}\right)$, since $\bar{D}(W) \cong \Lambda^{2}\left(U^{\text {odd }}\right)$. So choose an element $x_{i} \in W \cdot \Lambda\left(U^{\text {odd }}\right)$, such that $\bar{D}\left(x_{i}\right)=\bar{D}\left(v_{i}\right)$, for each $i$. Finally, set $\phi\left(v_{i}\right)=v_{i}-x_{i}$ and extend to an algebra map. Notice that $\phi$ makes the following diagram of extension sequences commute:

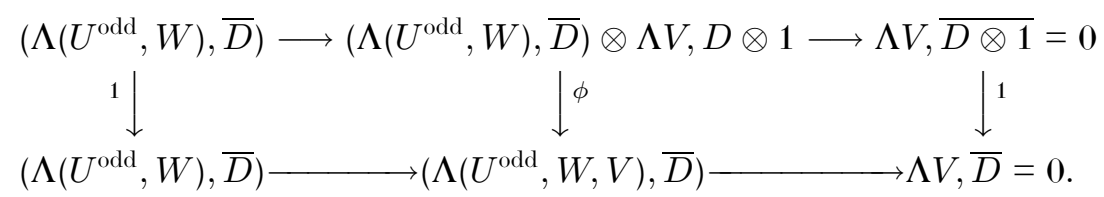

It follows that $\phi$ is a quasi-isomorphism. 
Returning to the inequality (5) displayed above, we now have

$$
\operatorname{dim} H(\Lambda(U, V)) \cdot 2^{\operatorname{dim} \bar{W}} \cdot 2^{\operatorname{dim} W} \geqslant \operatorname{dim} H\left(\Lambda\left(U^{\text {odd }}, W\right)\right) \cdot 2^{\operatorname{dim} V} .
$$

But $\operatorname{dim} H\left(\Lambda\left(U^{\text {odd }}, W\right)\right) \geqslant 2^{\operatorname{dim} W}$, by Proposition $2 \cdot 1$. Since $\operatorname{dim} \bar{W}=\operatorname{dim} U^{\text {even }}$, the result follows.

We can establish Conjecture $1 \cdot 1$ in several cases using Theorem $2 \cdot 3$. We begin with a discussion of two previously-known cases of Conjecture 1.1. We include these cases here to illustrate that Theorem $2 \cdot 3$ can be used to unify them with other results. Also, we include a self-contained proof in the second case that uses techniques from well within rational homotopy theory.

\subsection{The pure case}

For any elliptic minimal model $\Lambda W$, the homotopy Euler characteristic is $\chi_{\pi}:=$ $\operatorname{dim}\left(W^{\text {even }}\right)-\operatorname{dim}\left(W^{\text {odd }}\right)$. For any elliptic space, a result due to Allday and Halperin [AH, theorem 1] asserts that the rational toral rank is less than or equal to the negative of the homotopy Euler characteristic, thus $\operatorname{rk}_{0}(X) \leqslant-\chi_{\pi}$.

Now consider the so-called pure case, in which $\Lambda(U, V)$ is a two-stage, elliptic minimal model with $U=U^{\text {even }}$ and $V=V^{\text {odd }}$. In this case, Theorem $2 \cdot 3$ specializes to: $\operatorname{dim} H(\Lambda(U, V)) \geqslant 2^{-\chi_{\pi}}$. Combining these observations, we retrieve the following result of Halperin:

Corollary $2 \cdot 4$ ([Hal, proposition $1 \cdot 5])$. Let $X$ be an elliptic space with a pure minimal model. Then Conjecture $1 \cdot 1$ holds for $X$, i.e., $\operatorname{dim} H(X ; \mathbb{Q}) \geqslant 2^{\mathrm{rk}_{0}(X)}$.

We remark that this includes the case in which $X$ is a homogeneous space. Indeed, our proof of Theorem $2 \cdot 3$ incorporates the argument used by Halperin to obtain Conjecture $1 \cdot 1$ in the homogeneous space case.

\section{$2 \cdot 3$. The case of odd generators with quadratic differential (coformal spaces)}

We say that a minimal model is coformal if it has a quadratic differential, that is, if $d: W \rightarrow \Lambda^{2} W$. Notice that this really means quadratic with respect to a particular choice of generators for $\Lambda W$. A space is coformal if it has a coformal minimal model, and in this case its rational homotopy type is completely determined by its rational homotopy Lie algebra, the bracket of which is dual to $d$ [FHT, section 21].

The following result is due to Allday and Puppe:

Theorem $2 \cdot 5$ ([APa, proposition $3 \cdot 1$, corollary $3 \cdot 5])$. Let $X$ be a simply-connected, coformal elliptic space that has a two-stage minimal model $\Lambda(U, V)$ with odd-degree generators only. Assume that the two-stage decomposition displays $V$ with maximal dimension. Then, $r k_{0}(X)=\operatorname{dim} V$ and Conjecture $1 \cdot 1$ holds for $X$.

Proof. The equality $\operatorname{rk}_{0}(X)=\operatorname{dim} V$ is given in [APa, corollary 3.5]. (Actually, the rational toral rank is identified there with the dimension of the centre of the homotopy Lie algebra of $X$. However, standard ideas from rational homotopy theory can be used to identify $\operatorname{dim} V$ and the dimension of the centre of the homotopy Lie algebra, in this particular case.) Since the minimal model has odd-degree generators only, Theorem $2 \cdot 3$ specializes to the inequality $\operatorname{dim} H(\Lambda(U, V)) \geqslant 2^{\operatorname{dim} V}$.

This paper contains a self-contained proof of Theorem 2.5 that uses only standard tools from rational homotopy theory. For this, we need to supply our own argument 
for the equality $\operatorname{rk}_{0}(X)=\operatorname{dim} V$, to substitute for [APa, corollary 3.5]. This is obtained by combining Lemma 2.12 with Theorem 2.9 below, both of which apply to more general settings (cf. Remarks $2 \cdot 14$ ). When we prove Theorem $2 \cdot 9$, we will be more specific as to what the phrase 'displays $V$ with maximal dimension' entails.

We remark that Allday-Puppe conclude their result by appealing to a result of Deninger-Singhof [DS]. Indeed, the result from [DS] used to complete their argument is precisely a special case of Theorem $2 \cdot 3$, in which the differential is assumed to be quadratic and all generators are assumed to be of odd degree. It is therefore natural to ask whether $\operatorname{rk}_{0}(\Lambda(U, V))=\operatorname{dim} V$ more generally, at least in the case in which the two-stage, elliptic minimal model has odd-degree generators only. This is not true without some further hypotheses, as we illustrate in Example $3 \cdot 2$. We return to this point, and related questions, in the last section of the paper.

\subsection{New cases}

In the remainder of this section, we prove new results that give further situations to which we can apply Theorem $2 \cdot 3$. In phrasing our results, we must be careful about a technical point, namely, the choice of a two-stage decomposition that displays $V$ with maximal dimension. We illustrate this with an example.

Example 2.6. Let $\Lambda(U, V ; d)$ be a two-stage minimal model with bases $U=$ $\left\langle u_{1}, u_{2}, u_{3}\right\rangle$ and $V=\left\langle v_{1}\right\rangle$, all generators being of degree 1 . Set $d v_{1}=u_{1} u_{3}-u_{1} u_{2}+u_{2} u_{3}$, then each basis element of $U$ certainly occurs in a non-trivial differential. However, $V$ is not maximal here. We can see this by re-writing $d v_{1}=\left(u_{1}+u_{2}\right)\left(u_{1}+u_{3}\right)$, and noting that only 2 linearly independent elements of $U$ actually occur. In other words, we can change the basis in $U$, and move the 'spare' generator of $U$ into $V$. Specifically, define $\phi\left(u_{1}\right)=u_{1}, \phi\left(u_{2}\right)=u_{2}+u_{1}, \phi\left(u_{3}\right)=u_{3}+u_{1}$ and $\phi\left(v_{1}\right)=v_{1}$, then extend to an algebra automorphism of $\Lambda(U, V)$. If we set $d^{\prime}=\phi^{-1} d \phi$, then $\phi$ becomes an isomorphism of minimal models $\phi: \Lambda\left(U, V ; d^{\prime}\right) \rightarrow \Lambda(U, V ; d)$. A simple check reveals that $\Lambda\left(U, V ; d^{\prime}\right)$ is two-stage with $d^{\prime}\left(v_{1}\right)=u_{2} u_{3}$. Thus we can write it as $\Lambda\left(U^{\prime}, V^{\prime} ; d^{\prime}\right), U^{\prime}=\left\langle u_{2}, u_{3}\right\rangle$, and $V^{\prime}=\left\langle v_{1}, u_{1}\right\rangle$. In this latter case, $V^{\prime}$ is now of maximal dimension.

Definition $2 \cdot 7$. Suppose $(\Lambda(U, V), d)$ is a two-stage minimal model. We say that $V$ has maximal dimension, or that the two-stage decomposition displays $V$ with maximal dimension, if, for any isomorphic two-stage minimal model $\left(\Lambda\left(U^{\prime}, V^{\prime}\right), d^{\prime}\right) \cong$ $(\Lambda(U, V), d)$, we have $\operatorname{dim} V^{\prime} \leqslant \operatorname{dim} V$.

Since we assume that the spaces of generators are finite-dimensional, it is clear that every two-stage minimal model has a decomposition that displays $V$ with maximal dimension. Also, for the two-stage decomposition to display $V$ with maximal dimension, then it is clearly necesary (but not sufficient) that every generator from $U$ appear in some differential.

We now give a consequence of the two-stage decomposition being chosen so as to display $V$ of maximal dimension. This result does not cover all two-stage cases, but is sufficient for our purposes. We focus on the case in which the differential is quadratic. For parity of degree reasons, in this case we have $d(V) \subseteq \Lambda^{2}\left(U^{\text {odd }}\right) \oplus \Lambda^{2}\left(U^{\text {even }}\right)$. Let $U^{\text {odd }}=\left\langle u_{1}, \ldots, u_{p}\right\rangle, U^{\text {even }}=\left\langle w_{1}, \ldots, w_{q}\right\rangle$ and $V=\left\langle v_{1}, \ldots, v_{r}\right\rangle$ denote bases. From now on, we will adopt this as standard notation, for the case of a two-stage minimal model that may contain even-degree generators. 
Since we assume the differential is quadratic, for each basis element $u_{k}$ of $V$, we may write

$$
d v_{k}=\sum_{1 \leqslant i<j \leqslant p} a_{i, j}^{k} u_{i} u_{j}+\sum_{1 \leqslant i \leqslant j \leqslant q} b_{i, j}^{k} w_{i} w_{j}
$$

Using the coefficients from the first of these sums, for $k=1, \ldots, r$, we define the skew-symmetric, $p \times p$ matrix $M^{k}$, by

$$
M_{i, j}^{k}= \begin{cases}a_{i, j}^{k} & \text { if } i<j \\ -a_{i, j}^{k} & \text { if } i>j \\ 0 & \text { if } i=j\end{cases}
$$

Now, let $M=\left[\begin{array}{c}M^{1} \\ \vdots \\ M^{r}\end{array}\right]$ be the $p r \times p$ block marix formed by the $M^{k}$ as rows.

Lemma 2.8. If $V$ has maximal dimension in $\Lambda(U, V)$, then rank $M=p$. In particular, there is a $p \times r p$ matrix $N$ such that $N M$ is the $p \times p$ identity matrix.

Proof. Let $U^{*}$ denote the dual space of $U$, and for $u^{*} \in U^{*}$, let $i_{u^{*}}$ denote the derivation of $\Lambda(U, V)$ of degree -1 extending the linear map $i_{u^{*}}: U \oplus V \rightarrow \mathbb{Q}$ defined by $i_{u^{*}}(x+y)=u^{*}(x)$, for $x \in U, y \in V$. We first show that, under our hypotheses, the Lie derivative $\mathcal{L}: U^{*} \rightarrow \operatorname{Hom}(V, U)$ defined by

$$
\mathcal{L}\left(u^{*}\right)=i_{u^{*}} d-(-1)^{\left|u^{*}\right|} d i_{u^{*}}=i_{u^{*}} d
$$

is injective. (In fact, the injectivity of $\mathcal{L}$ is equivalent to the maximality of $\operatorname{dim} V$.) Let $K=\operatorname{ker} \mathcal{L}$ and choose a complement $X \subset U^{*}$ for $K$ so that $U^{*}=K \oplus X$ and $U=K^{\perp} \oplus X^{\perp}$, where, if $W \subset U^{*}, W^{\perp}=\left\{u \in U \mid \forall_{w} \in W, w(u)=0\right\}$. Then, as

$$
\Lambda U=\left(\Lambda X^{\perp} \otimes \Lambda X^{\perp}\right) \oplus\left(\Lambda X^{\perp} \otimes \Lambda^{+} K^{\perp}\right) \oplus\left(\Lambda^{+} K^{\perp} \otimes \Lambda^{+} K^{\perp}\right),
$$

the definition of $K$ shows that $d V \subset A^{+} K^{\perp} \otimes \Lambda^{+} K^{\perp}$. Now define $U_{1}=K^{\perp}$ and $V_{1}=V \oplus X^{\perp}$, with $d_{U_{1}}^{\prime}=0, d_{\mid V}^{\prime}=d_{\mid V}$ and $d_{X^{\perp}}^{\prime}=0$. Then $U_{1} \oplus V_{1}=U \oplus V$, and this induces an isomorphism of two-stage minimal models $\Lambda\left(U_{1}, V_{1} ; d^{\prime}\right) \cong \Lambda(U, V ; d)$. Since $V$ has maximal dimension, $K$ must be 0 .

Let $\left\{u_{1}, \ldots, u_{p}\right\}$ be a basis of $U^{\text {odd }}$, and $\left\{u_{1}^{*}, \ldots, u_{p}^{*}\right\}$ the dual basis. Then, as $V$ has maximal dimension, we know that in particular the maps $\mathcal{L}\left(u_{j}^{*}\right)=i_{u_{j}^{*}} d: V \rightarrow U^{\text {odd }}$ for $j=1, \ldots, p$ are linearly independent. In other words, if $c=\left(c_{1}, \ldots, c_{p}\right)^{t} \in \mathbb{Q}^{p}$, then $\sum_{j} c_{j} i_{u_{j}^{*}} d v_{k}=\sum_{i, j} M_{i, j}^{k} u_{i} c_{j}=0$ for $k=1, \ldots, r$, implies that $c=0$. Because the $u_{i}$ are linearly independent, this is equivalent to the statement

$$
\sum_{j} M_{i, j}^{k} c_{j}=0 \quad \text { for } i=1, \ldots, p \text { and } k=1, \ldots, r \Longrightarrow c=0 .
$$

That is, $M c=0 \Longrightarrow c=0$. Hence, $\operatorname{rank} M=p$.

To phrase our results, we use the following terminology: we say that a graded vector space $V$ is $n$-co-connected if $V^{i}=0$ for $i \geqslant n$.

Theorem 2.9. Let $\Lambda(U, V)$ be an elliptic, two-stage minimal model. Assume that $d V \subseteq$ $\Lambda^{2} U$, and that the two-stage decomposition displays $V$ with maximal dimension. Finally, 
assume that $U^{\text {odd }}$ and $U^{\text {even }}$ satisfy one of the following connectivity and co-connectivity hypotheses:

(A) $U^{\text {odd }} i s(2 r-1)$-connected and $U^{\text {even }} i s(2 r+2)$-co-connected;

(B) $U^{\text {odd }}$ is $(2 s+1)$-co-connected and $U^{\text {even }} i s(4 s-4)$-connected.

Then $\operatorname{rk}_{0}(\Lambda(U, V)) \leqslant \operatorname{dim} V-\operatorname{dim} U^{\text {even }}$.

Proof. If $\operatorname{rk}_{0}(\Lambda(U, V))=n$, then we have a K-S-extension $\left(\Lambda A_{n} \otimes \Lambda(U, V), D\right)$ as in (1) that has finite-dimensional cohomology. We claim that in any such minimal model, we can assume that $D\left(U^{\text {odd }}\right)$ is contained in $I\left(U^{\text {odd }} \oplus V\right)$, the ideal in $\Lambda A_{n} \otimes \Lambda(U, V)$ generated by $U^{\text {odd }} \oplus V$.

Allowing this claim for now, we appeal to some results of Halperin concerning elliptic minimal models. First of all, to any elliptic minimal model $(\Lambda W, d)$, there is an associated pure model, denoted $\left(\Lambda W, d_{\sigma}\right)$, which is defined by adjusting the differential $d$ to $d_{\sigma}$ as follows: we set $d_{\sigma}=0$ on each even degree generator of $W$, and on each odd degree generator $w \in W$, we set $d_{\sigma}(w)$ equal to the part of $d(w)$ contained in $\Lambda\left(W^{\text {even }}\right)$. One checks that this defines a differential $d_{\sigma}$ on $\Lambda W$, and thus we obtain a pure (minimal) model $\left(\Lambda W, d_{\sigma}\right)$. Then, by [Hala, proposition 1] (ef. also [FHT, proposition 32.4]), $\operatorname{dim} H(\Lambda W, d)$ is finite-dimensional if and only if $\operatorname{dim} H\left(\Lambda W, d_{\sigma}\right)$ is finite-dimensional. Applying all this to the minimal model $\left(\Lambda A_{n} \otimes \Lambda(U, V)\right.$, $\left.D\right)$, we obtain the following.

From the claim, $\left(\Lambda A_{n} \otimes \Lambda(U, V), D_{\sigma}\right)$ satisfies $D_{\sigma}\left(U^{\text {odd }}\right)=0$, and therefore $\left(\Lambda A_{n} \otimes\right.$ $\left.\Lambda(U, V), D_{\sigma}\right) \cong\left(\Lambda U^{\text {odd }}, D_{\sigma}=0\right) \otimes\left(\Lambda A_{n} \otimes \Lambda\left(U^{\text {even }}, V\right), D_{\sigma}\right)$. Since $\left(\Lambda A_{n} \otimes \Lambda(U, V), D_{\sigma}\right)$ has finite-dimensional cohomology, so does $\left(\Lambda A_{n} \otimes \Lambda\left(U^{\text {even }}, V\right), D_{\sigma}\right)$. It follows that $\left(\Lambda A_{n} \otimes \Lambda\left(U^{\text {even }}, V\right), D_{\sigma}\right)$ is an elliptic minimal model, and therefore that its homotopy Euler characteristic is non-positive [Hala, theorem 1] (cf. also [FHT, proposition $32 \cdot 10]$ ). This implies that $n \leqslant \operatorname{dim} V-\operatorname{dim} U^{\text {even }}$, as required.

It only remains to establish the claim. We do this by a careful analysis of the terms that can occur in the differentials. Our argument is essentially the same as that in [APb, theorem 4.6]. First we write, for each $u_{k}$ in the basis of $V$,

$$
D v_{k}=\sum_{1 \leqslant i<j \leqslant p} a_{i, j}^{k} u_{i} u_{j}+\sum_{1 \leqslant i \leqslant j \leqslant q} b_{i, j}^{k} w_{i} w_{j}+\text { terms in the ideal } I\left(A_{n}\right) .
$$

The coefficients $a_{i, j}^{k}$ and $b_{i, j}^{k}$ are scalars. Our strategy in either case (A) or case (B) is the same. We apply $D$ to $(7)$, obtaining $D^{2} v_{k}=0$ on the left-hand side. By focussing on certain terms present on the right-hand side, we obtain sufficient information to complete our argument.

Consider hypothesis (A) first. Here, from the (co-)connectivity restrictions, we see that $D\left(U^{\text {even }}\right) \subseteq \Lambda^{+} A_{n} \otimes \Lambda U^{\text {even }} \otimes \Lambda^{+} V$. Therefore, display the terms in (7) as follows. Write

$$
\begin{aligned}
D v_{k}= & \sum_{1 \leqslant i<j \leqslant p} a_{i, j}^{k} u_{i} u_{j}+\sum_{1 \leqslant i<j \leqslant p} P_{i, j}^{k} u_{i} u_{j}+\sum_{\substack{i=1, \ldots, r \\
j=1, \ldots, p}} R_{i, j}^{k} u_{i} u_{j} \\
& +P_{k}+\text { terms in the ideal } I\left(\Lambda^{2}(V) \oplus \Lambda^{\geqslant 4}\left(U^{\text {odd }}, V\right)\right) .
\end{aligned}
$$

Here, $P_{k}$ includes all terms from $\Lambda A_{n} \otimes \Lambda\left(U^{\text {even }}\right)$ present in (7), and the coefficients $P_{i, j}^{k}$ and $R_{i, j}^{k}$ denote terms in $\Lambda^{+} A_{n}$. After applying $D$ to this, we will consider only contributions in $\left(\Lambda^{+} A_{n} \otimes \Lambda\left(U^{\text {even }}\right)\right) \cdot U^{\text {odd }}$. Define a derivation $\delta$ of $\Lambda A_{n} \otimes \Lambda(U, V)$ as follows. On generators from $U^{\text {odd }} \quad V$, set $\delta$ equal to the part of $D$ : $U^{\text {odd }} \quad V$ 
$\Lambda A_{n} \otimes \Lambda(U, V)$ whose image is contained in $\Lambda^{+} A_{n} \otimes \Lambda\left(U^{\text {even }}\right)$. On the remaining generators, set $\delta\left(U^{\text {even }}\right)=0$. Extend $\delta$ to a derivation on $\Lambda\left(A_{n}, U, V\right)$. Applying $D$ to (8), and collecting terms in $\left(\Lambda^{+} A_{n} \otimes \Lambda\left(U^{\text {even }}\right)\right) \cdot U^{\text {odd }}$, we have

$$
0=\delta\left(\sum_{1 \leqslant i<j \leqslant p} a_{i, j}^{k} u_{i} u_{j}\right)+\delta\left(\sum_{1 \leqslant i<j \leqslant p} P_{i, j}^{k} u_{i} u_{j}\right)+\sum_{\substack{i=1, \ldots, r \\ j=1, \ldots, p}} \delta\left(R_{i, j}^{k} v_{i}\right) u_{j} .
$$

We fix an index $l$, with $1 \leqslant l \leqslant p$, and collect all coefficients of $u_{l}$ from this equation, to obtain

$$
0=\sum_{i<l} a_{i, l}^{k} \delta\left(u_{i}\right)-\sum_{l<j} a_{l, j}^{k} \delta\left(u_{j}\right)+\sum_{i<l} \delta\left(P_{i, l}^{k} u_{i}\right)-\sum_{l<j} \delta\left(P_{l, j}^{k} u_{j}\right)+\sum_{i=1, \ldots, r} \delta\left(R_{i, l}^{k} v_{i}\right) .
$$

Since $P_{i, j}^{k}, R_{i, j}^{k} \in \Lambda^{+} A_{n}$, and since $M^{k}$ is skew-symmetric, we obtain

$$
\sum_{i \neq l} a_{l, i}^{k} \delta\left(u_{i}\right)=\delta\left(\chi_{k, l}\right)
$$

for some element $\chi_{k, l} \in \Lambda^{+} A_{n} \cdot(U \oplus V)$. Let $\mathbf{u}=\left(u_{1}, \ldots, u_{p}\right)^{t}, \sigma_{k}=\left(\chi_{k, 1}, \ldots, \chi_{k, p}\right)$, and $\chi=\left(\sigma_{1}, \ldots, \sigma_{r}\right)^{t}$, and let $\delta$ act component-wise. Then the previous equation can be restated as

$$
\delta(M \mathbf{u})=\delta \chi .
$$

Now we apply Lemma $2 \cdot 8$ to obtain a $p \times r p$ matrix $N$ such that

$$
\delta \mathbf{u}=\delta(N M \mathbf{u})=\delta(N \chi) .
$$

Finally, we observe that this implies for each basis element of $U^{\text {odd }}$, we have $\delta\left(u_{i}\right)=$ $\delta\left(\chi_{i}\right)$ for some decomposable $\chi_{i} \in \Lambda^{+} A_{n} \cdot\left(U^{\text {odd }} \oplus V\right)$. Returning to the full differential $D$, we rephrase this as follows: for each basis element $u_{i}$ of $U^{\text {odd }}$, there is some $\chi_{i} \in \Lambda^{+} A_{n} \cdot\left(U^{\text {odd }} \oplus V\right)$ for which $D\left(u_{i}-\chi_{i}\right) \in I\left(U^{\text {odd }}, V\right)$.

As in Example 2.6, we now change the basis in $U^{\text {odd }}$. Define an isomorphism of algebras $\phi: \Lambda A_{n} \otimes \Lambda(U, V) \rightarrow \Lambda A_{n} \otimes \Lambda(U, V)$ on generators by setting $\phi\left(u_{i}\right)=u_{i}-\chi_{i}$ for each basis element $u_{i}$ of $U^{\text {odd }}$, and $\phi=$ id on generators of $A_{n}, U^{\text {even }}$ and $V$. Then extend $\phi$ to an algebra isomorphism. Now define a differential by $D^{\prime}=\phi^{-1} D \phi$, so that $\phi$ becomes an isomorphism of minimal models $\phi:\left(\Lambda A_{n} \otimes \Lambda(U, V), D^{\prime}\right) \rightarrow\left(\Lambda A_{n} \otimes\right.$ $\Lambda(U, V), D)$. An easy check now confirms that $D^{\prime}\left(A_{n}\right)=0$ and $D^{\prime}\left(U^{\text {odd }}\right) \subseteq I\left(U^{\text {odd }}, V\right)$, as desired.

A similar argument is used under hypothesis (B). Here, if some coefficient $a_{i, j}^{k} \neq 0$ in (7), then the degree of $D v^{k}$ must be at most $4 s-2$. But under hypothesis (B), terms in $\Lambda^{2}\left(U^{\text {even }}\right)$ start in degree $8 s-4$ and terms in $\Lambda^{+} A_{n} \cdot \Lambda U^{\text {even }}$ start in degree $4 s$. In this case, therefore, whenever some $a_{i, j}^{k} \neq 0$, then the remaining terms of $(7)$ do not include any in the ideal $I\left(U^{\text {even }}\right)$. So suppose that $v^{k}$ is of suitable degree for $D\left(v^{k}\right)$ to contain non-zero terms $a_{i, j}^{k} u_{i} u_{j}$. Display the terms in (7) as follows. Write

$$
\begin{aligned}
D\left(v_{k}\right)= & \sum_{1 \leqslant i<j \leqslant p} a_{i, j}^{k} u_{i} u_{j}+\sum_{1 \leqslant i<j \leqslant p} P_{i, j}^{k} u_{i} u_{j}+\sum_{\substack{i=1, \ldots, r \\
j=1, \ldots, p}} R_{i, j}^{k} v_{i} u_{j} \\
& +P_{k}+\text { terms in } I\left(\Lambda^{\geqslant 2} V \oplus \Lambda^{\geqslant 4} U^{\text {odd }}\right),
\end{aligned}
$$

where $P_{k}$ denotes a term from $\Lambda^{+} A_{n}$ and the coefficients $P_{i, j}^{k}$ and $R_{i, j}^{k}$ denote terms in 
$\Lambda^{+} A_{n}$. After applying $D$ to this, we will consider only contributions in $\left(\Lambda^{+} A_{n}\right) \cdot U^{\text {odd }}$. To this end, define a derivation $\delta$ of $\Lambda A_{n} \otimes \Lambda(U, V)$ as follows. On generators from $U^{\text {odd }} \otimes V$, set $\delta$ equal to the part of $D: U^{\text {odd }} \oplus V \rightarrow \Lambda A_{n} \otimes \Lambda(U, V)$ whose image is contained in $\Lambda^{+} A_{n}$. On the remaining generators, set $\delta\left(U^{\text {even }}\right)=0$. Applying $D$ to $(9)$, and collecting terms in $\left(\Lambda^{+} A_{n}\right) \cdot U^{\text {odd }}$ yields

$$
0=\delta\left(\sum_{1 \leqslant i<j \leqslant p} a_{i, j}^{k} u_{i} u_{j}\right)+\delta\left(\sum_{1 \leqslant i<j \leqslant p} P_{i, j}^{k} u_{i} u_{j}\right)+\sum_{\substack{i=1, \ldots, r \\ j=1, \ldots, p}} \delta\left(R_{i, j}^{k} v_{i}\right) u_{j},
$$

for each index $k$ that has at least one coefficient $a_{i, j}^{k}$ non-zero. From here, the argument proceeds exactly as that for hypothesis (A).

This completes the proof of the claim, under either hypothesis. By the first part of the proof, the result follows.

There are other situations in which a similar approach gives an upper bound on the toral rank. We give one more result that moves away from the quadratic differential restriction.

Theorem 2.10. Let $\Lambda(U, V)$ be a two-stage minimal model with odd degree generators only and assume that the two-stage decomposition displays $V$ with maximal dimension. Suppose there are positive integers $r$ and $s$, with $r \leqslant s \leqslant 2 r$, for which $U=U^{\text {odd }} i s(r-1)$ connected and $(s+1)$-co-connected. Suppose further that there are positive integers $t$ and $u$, with $s \leqslant t \leqslant u \leqslant s+r$, for which $V$ is $(t-1)$-connected and $(u+1)$-co-connected. Then $\operatorname{rk}_{0}(\Lambda(U, V ; d)) \leqslant \operatorname{dim} V$.

Proof. If $\operatorname{rk}_{0}(\Lambda(U, V ; d))=n$, then we have an extension $\left(\Lambda A_{n} \otimes \Lambda(U, V), D\right)$ with finite-dimensional cohomology. In such an extension, from the assumptions on the degrees of the generators, we must have $D u_{i} \in \Lambda A_{n}$ for each generator $u_{i}$ of $U$. We claim that in fact $D u_{i}=0$. Suppose the shortest length of any non-zero term that occurs in some polynomial $D u_{i}$ is $l$. Then as in the proof of Theorem $2 \cdot 9$, define a derivation $\delta$ on $U$ by setting $\delta u_{i}$ equal to the term of length $l$ that occurs in $D u_{i}$, or zero if there is no such term. Define $\delta$ to be zero on all generators of $A_{n}$ and $V$, and extend as a derivation to $\Lambda A_{n} \otimes \Lambda(U, V)$. Without loss of generality, we can suppose that the non-zero terms $\delta u_{i}$ are linearly independent. Otherwise, we can change basis within $U$ to make these so. Next, consider $D v$ for a generator in $V$. We have $D v=d v+\chi$, for $d v \in \Lambda U$ and some $\chi$ in the ideal of $\Lambda A_{n} \otimes \Lambda(U, V)$ generated by $A_{n}$. The (co)-connectivity hypotheses on $V$ imply that $D v \in \Lambda A_{n} \otimes \Lambda U$, for any $v \in V$. Therefore, $\chi \in \Lambda^{+} A_{n} \otimes \Lambda U$ and hence $D(\chi) \in A^{>l} A_{n} \otimes \Lambda U$. Consequently, when we equate terms in $\Lambda^{l} A_{n} \otimes \Lambda U$ that arise in the equation $0=D^{2} v=D(d v)+D(\chi)$, we have $0=\delta(d v)$. By the assumption that non-zero $\delta u_{i}$ 's are linearly independent, it follows from an easy argument that $\delta u_{i}=0$ for each $u_{i}$ that occurs in the differential $d v$. Finally, our assumption that $V$ is taken as large as possible implies that each generator $u_{i}$ does occur in at least one differential $d v$. This implies that the shortest length terms $\delta u_{i}$ are zero, and an induction completes the proof of our claim, namely that $D u_{i}=0$ for each $u_{i} \in U$.

So far, we have argued that in any extension $\left(\Lambda A_{n} \otimes \Lambda(U, V), D\right)$, our hypotheses imply that $D(U)=0$. Now an argument as in the second paragraph of the proof of Theorem 29 shows that $n \leqslant \operatorname{dim} V$. 
Corollary 2.11. Let $X$ be a simply connected, elliptic space with two-stage minimal model. If the minimal model satisfies either the hypotheses of Theorem 2.9, or those of Theorem 2.10, then Conjecture 1·1 holds for $X$.

Proof. Combine Theorem $2 \cdot 3$ with the results mentioned in the statement.

It is natural to ask whether $\operatorname{rk}_{0}(X) \leqslant \operatorname{dim} V-\operatorname{dim} U^{\text {even }}$ for a general two-stage, elliptic space. This is not the case in general, as we illustrate in Example $3 \cdot 1$ below. Indeed, under hypotheses that include all cases in which the two-stage minimal model has odd-degree generators only, we can actually reverse the direction in this inequality.

Lemma 2.12. Suppose $\Lambda U \otimes \Lambda V$ is an elliptic two-stage minimal model with $U^{\text {even }}=$ $U^{2 n}$ for some fixed $n \in \mathbb{N}$. Then, $\operatorname{rk}_{0}(\Lambda U \otimes \Lambda V) \geqslant \operatorname{dim} V-\operatorname{dim} U^{\text {even }}$.

Proof. Consider the extension sequence

$$
\left(\Lambda U^{\text {odd }}, 0\right) \longrightarrow\left(\Lambda U^{\text {odd }} \otimes \Lambda U^{\text {even }} \otimes \Lambda V, d\right) \longrightarrow\left(\Lambda U^{\text {even }} \otimes \Lambda V, \bar{d}\right) .
$$

Since $V=V^{\text {odd }}$ the right-hand term is now pure (and elliptic) with $U^{\text {even }}=U^{2 n}$. By [Jes, lemma 3·3], there is an isomorphism of two-stage models

$$
\rho:\left(\Lambda U^{\text {even }} \otimes \Lambda V, \bar{d}\right) \longrightarrow\left(\Lambda U^{\text {even }} \otimes \Lambda\left(V^{\prime} \oplus V^{\prime \prime}\right), \bar{d}^{\prime}\right)
$$

such that

(1) $\operatorname{dim} H\left(\Lambda U^{\text {even }} \otimes V^{\prime}, \bar{d}\right)<\infty$,

(2) $\operatorname{dim} V^{\prime}=\operatorname{dim} U^{\text {even }}$,

(3) $\rho$ is the identity on $U^{\text {even }}$, and

(4) $\rho(V) \subset V^{\prime} \oplus V^{\prime \prime} \oplus\left(\Lambda^{+} U^{\text {even }} \otimes\left(V^{\prime} \oplus V^{\prime \prime}\right)\right)$.

These conditions imply that $\rho$ induces an isomorphism $V \cong V^{\prime} \oplus V^{\prime \prime}$, and that $\rho^{-1}\left(V^{\prime} \oplus V^{\prime \prime}\right) \subset V \oplus\left(\Lambda^{+} U^{\text {even }} \otimes V\right)$. We now extend $\rho$ to an isomorphism of algebras

$$
\tilde{\rho}: \Lambda U \otimes \Lambda V \longrightarrow \Lambda U \otimes \Lambda\left(V^{\prime} \oplus V^{\prime \prime}\right)
$$

by letting it be the identity on $\Lambda U$. Define a derivation $d^{\prime}$ on $\Lambda U \otimes \Lambda\left(V^{\prime} \oplus V^{\prime \prime}\right)$ by setting $d^{\prime} U=0$, and $d^{\prime} v^{\prime}=d \rho^{-1}\left(v^{\prime}\right)$ for $v^{\prime} \in\left(V^{\prime} \oplus V^{\prime \prime}\right)$. In order to show that

$$
\tilde{\rho}:(\Lambda U \otimes \Lambda V, d) \longrightarrow\left(\Lambda U \otimes \Lambda\left(V^{\prime} \oplus V^{\prime \prime}\right), d^{\prime}\right)
$$

is an isomorphism of models, it suffices to show that $\left(d^{\prime}\right)^{2} v^{\prime}=0$ for $v^{\prime} \in V^{\prime} \oplus V^{\prime \prime}$, so we compute:

$$
\begin{aligned}
\left(d^{\prime}\right)^{2} v^{\prime} & =d^{\prime}\left(d \rho^{-1}\left(v^{\prime}\right)\right) \\
& \in d^{\prime}\left(d\left(V \oplus\left(\Lambda^{+} U^{\text {even }} \otimes V\right)\right)\right) \\
& \subset d^{\prime}(\Lambda U) \\
& =\{0\} .
\end{aligned}
$$

Thus, we may assume that $V=V^{\text {odd }}=V^{\prime} \oplus V^{\prime \prime}$, with $\operatorname{dim} H\left(\Lambda U \otimes \Lambda V^{\prime}\right)<\infty$, and $\operatorname{dim} V^{\prime}=\operatorname{dim} U^{\text {even }}$. Now suppose $V^{\prime \prime}=\left\langle v_{1}, \ldots, v_{n}\right\rangle$, and define a K-S-extension

$$
\left(\Lambda A_{n}, 0\right) \longrightarrow\left(\Lambda A_{n} \otimes \Lambda(U, V), D\right) \longrightarrow(\Lambda(U, V), d)
$$

in which $A_{n}=\left\langle a_{1}, \ldots, a_{n}\right\rangle$ with $\left|a_{i}\right|=2$, by $D v_{i}=d v_{i}+a_{i}^{\frac{\left|v_{i}\right|+1}{2}}$, and $d=D$ otherwise. A standard argument (using the associated pure model, as in the proof of Theorem 29 ), 
now shows that $\operatorname{dim} H\left(\Lambda A_{n} \otimes \Lambda(U, V), D\right)$ is finite-dimensional, so that $\operatorname{rk}_{0} \Lambda(U, V) \geqslant$ $n=\operatorname{dim} V^{\prime \prime}=\operatorname{dim} V-\operatorname{dim} U^{\text {even }}$.

Consequently, we obtain values for the rational toral rank in the following cases.

Corollary 2.13. Let $X$ be a simply connected elliptic space with two-stage minimal model $\Lambda(U, V)$. Suppose that the two-stage decomposition displays $V$ with maximal dimension.

(1) If the minimal model has quadratic differential, satisfies one of the (co-) connectivity conditions of Theorem 2.9 and also satisfies $U^{\text {even }}=U^{2 n}$ for some fixed $n$, then $\operatorname{rk}_{0}(X)=\operatorname{dim} V-\operatorname{dim} U^{\text {even }}$.

(2) If the minimal model has odd-degree generators only, and satisfies the (co-) connectivity conditions of Theorem $2 \cdot 10$, then $\operatorname{rk}_{0}(X)=\operatorname{dim} V$.

Proof. Combine Lemma $2 \cdot 12$ with the results mentioned in the statement.

Remarks $2 \cdot 14$. We can specialize corollary $2 \cdot 13(1)$ in a number of interesting directions. For example, if $U^{\text {even }}=0$, then we retrieve the identification $\operatorname{rk}_{0}(X)=\operatorname{dim} V$ of Theorem $2 \cdot 5$. As a second example, we can restrict to the case in which $U^{\text {odd }}=0$. This is the case in which the minimal model is pure with quadratic differential, and also satisfies $U^{\text {even }}=U^{2 n}$ for some fixed $n$. Here, we obtain $\operatorname{rk}_{0}(X)=-\chi_{\pi}(X)$.

\section{Examples, comments and questions}

In this section we mention various examples and results. Our focus here is more on the exact toral rank, rather than the bound of Conjecture $1 \cdot 1$.

\section{$3 \cdot 1$. The relation between $\operatorname{rk}_{0}(X)$ and $\operatorname{dim} V-\operatorname{dim} U^{\text {even }}$}

We begin with the simplest example that we can find to illustrate that the inequality $\operatorname{rk}_{0}(X) \leqslant \operatorname{dim} V-\operatorname{dim} U^{\text {even }}$ does not hold in general for a two-stage, elliptic space with both even and odd generators.

Example 3.1. Consider the two-stage minimal model $\Lambda\left(u_{1}, u_{2}, u_{3}, u_{4}, u_{5}, u_{6}, w, v\right)$, with $\left|u_{1}\right|=\left|u_{2}\right|=\left|u_{3}\right|=3,\left|u_{4}\right|=7,\left|u_{5}\right|=23,\left|u_{6}\right|=27,|w|=18$ and $|v|=35$, and the single non-trivial differential given by $d v=w^{2}-u_{1} u_{2} u_{4} u_{5}-u_{1} u_{2} u_{3} u_{6}$. The associated pure model satisfied $\left(\Lambda(U, V), d_{\sigma}\right) \cong\left(\Lambda\left(u_{1}, u_{2}, u_{3}, u_{4}, u_{5}, u_{6}\right), d_{\sigma}=0\right) \otimes\left(\Lambda(w, v), d_{\sigma}\right)$, with $d_{\sigma}(w)=0$ and $d_{\sigma}(v)=w^{2}$. Now $H\left(\Lambda(w, v), d_{\sigma}\right) \cong \Lambda(w) /\left(w^{2}\right)$, so $(\Lambda(U, V)$, d) is elliptic.

The two-stage decomposition $U=\left\langle u_{1}, u_{2}, u_{3}, u_{4}, u_{5}, u_{6}, w\right\rangle$ and $V=\langle v\rangle$ displays $V$ with maximal dimension, so we have $\operatorname{dim} V-\operatorname{dim} U^{\text {even }}=0$. We now show that $\left.\operatorname{rk}_{0}(\Lambda(U, V))\right) \geqslant 1$. Let $a$ be a generator of degree 2 , and define a differential $D$ on $\Lambda a \otimes \Lambda(U, V)$ as follows: $D a=D u_{1}=D u_{2}=D u_{3}=D u_{4}=D w=0, D u_{5}=a^{3} w$, $D u_{6}=a^{2} w u_{1} u_{2}$ and $D v=w^{2}-u_{1} u_{2} u_{4} u_{5}-u_{1} u_{2} u_{3} u_{6}+a^{18}-a u_{6} u_{4}$. A straightforward check shows that this defines a differential. We show that $(\Lambda a \otimes \Lambda(U, V), D)$ has finite-dimensional cohomology. The associated pure model in this case is $\left(\Lambda a \otimes \Lambda(U, V), D_{\sigma}\right) \cong\left(\Lambda\left(u_{1}, u_{2}, u_{3}, u_{4}, u_{6}\right), D_{\sigma}=0\right) \otimes\left(\Lambda\left(a, w, u_{5}, v\right), D_{\sigma}\right)$, with $D_{\sigma} a=0$, $D_{\sigma}(w)=0, D \sigma u_{5}=a^{3} w$ and $D_{\sigma} v=w^{2}+a^{18}$. We observe that $D_{\sigma}\left(a^{3} v\right)=a^{3} w^{2}+$ $a^{21}=D_{\sigma}\left(u_{5} w\right)+a^{21}$, hence $D_{\sigma}\left(a^{3} v-u_{5} w\right)=a^{21}$, so [Hal, proposition 1] shows that $H\left(\Lambda a \otimes \Lambda(U, V), D_{\sigma}\right)$ is finite-dimensional. It follows that $H(\Lambda a \otimes \Lambda(U, V), D)$ is finite dimensional. Thus $\left.\operatorname{rk}_{0}(\Lambda(U, V))\right) \geqslant 1$. 
We assert that more work will show $\left.\operatorname{rk}_{0}(\Lambda(U, V))\right)=1$ in Example $3 \cdot 1$. We also remark that whilst Conjecture $1 \cdot 1$ does not follow from Theorem $2 \cdot 3$ in this example, it is nonetheless easily confirmed here.

Next, we specialize to the case of odd generators only. Unfortunately, this restriction alone does not give us the inequality $\operatorname{rk}_{0}(X) \leqslant \operatorname{dim} V$. The following example lies immediately outside the hypotheses of Theorem $2 \cdot 10$, at least as far the generators of $U$ are concerned.

Example 3.2. Let $\Lambda W=\Lambda(U, V)=\Lambda\left(u_{1}, u_{2}, u_{3}, u_{4}, u_{5}, v_{1}, v_{2}\right)$ be a two-stage minimal model with degrees and differential as follows: $\left|u_{1}\right|=\left|u_{2}\right|=\left|u_{3}\right|=\left|u_{4}\right|=3,\left|u_{5}\right|=7$, $\left|v_{1}\right|=9,\left|v_{2}\right|=11, d u_{i}=0$ for each $i, d v_{1}=u_{1} u_{5}$ and $d v_{2}=u_{1} u_{2} u_{3} u_{4}$. Then $\operatorname{dim} V=2$. We show that $\operatorname{rk}_{0}(\Lambda W) \geqslant 3$. First we describe an extension $\Lambda A \otimes \Lambda W, D$, with $A=\left\langle a_{1}, a_{2}, a_{3}\right\rangle$. Set $D=0$ on $\left\{a_{1}, a_{2}, a_{3}, u_{1}, u_{2}, u_{3}\right\}$, and $D u_{4}=-a_{1}^{2}, D u_{5}=a_{1} u_{1} u_{2}$, $D v_{1}=u_{1} u_{5}+a_{2}^{5}$ and $D v_{2}=u_{1} u_{2} u_{3} u_{4}+u_{3} u_{5} a_{1}+a_{3}^{6}$. A straightforward check shows that $D$ is a differential. We argue exactly as in the latter part of Example $3 \cdot 1$ to show that $H(\Lambda A \otimes \Lambda W, D)$ is finite-dimensional. It follows that $\operatorname{rk}_{0}(\Lambda W) \geqslant 3$.

Once again, Conjecture $1 \cdot 1$ does hold for Example $3 \cdot 2$, although we are unable to conclude this from our preceding results. To see this, use $[\mathbf{A P b}$, theorem 4.6$]$ to conclude $\operatorname{rk}_{0}(\Lambda W) \leqslant 5$. (In Example $3 \cdot 2$, the differential $d v_{1}=u_{1} u_{5}$ means that $u_{1}$ and $u_{5}$ correspond to non-central generators.) Moroever, the fibration with $u_{1}$ as base has fibre $\Lambda\left(u_{2}, u_{3}, u_{4}, u_{5}, v_{1}, v_{2}\right)$ with zero differential, and $\Lambda\left(u_{2}, u_{3}, u_{4}, u_{5}\right)$ is clearly in the kernel of the associated Wang derivation $\theta^{*}$. As in the proof of Theorem $2 \cdot 1$, we conclude that $\operatorname{dim} H(\Lambda W)=2 \operatorname{dim} \operatorname{ker} \theta^{*} \geqslant 2.2^{4}=2^{5} \geqslant 2^{\mathrm{rk}_{0}(\Lambda W)}$.

Another example in which $\operatorname{rk}_{0}(X)>\operatorname{dim} V-\operatorname{dim} U^{\text {even }}$ is given in Example 3.3. On the other hand, we currently have no example of a two stage minimal model for which $\mathrm{rk}_{0}(X)<\operatorname{dim} V-\operatorname{dim} U^{\text {even }}$. This leads us to wonder whether the hypotheses of Lemma $2 \cdot 12$ might be relaxed considerably. Note that in certain cases, the inequality $\operatorname{rk}_{0}(X) \geqslant \operatorname{dim} V-\operatorname{dim} U^{\text {even }}$ can be combined with other information to identify the toral rank, and we have seen instances of this in Corollary $2 \cdot 13$. As another example, whenever this latter inequality holds in a pure case, it identifies the toral rank as equal to $-\chi_{\pi}$ (cf. Remarks $\left.2 \cdot 14\right)$.

\subsection{Products}

Now we consider the question of how the toral rank behaves with respect to products. Although our previous results concerned the two-stage case, the comments here are not restricted to that case. It is easy to see that, in general, we have the inequality $\mathrm{rk}_{0}(X \times Y) \geqslant \mathrm{rk}_{0}(X)+\mathrm{rk}_{0}(Y)$. The following example shows that we may sometimes have strict inequality.

Example $3 \cdot 3$. Consider two-stage, elliptic minimal models $\mathcal{M}=\Lambda(x, y)$ with $|x|=$ $12,|y|=23$, and $d y=x^{2}$; and $\mathcal{N}=\Lambda\left(u_{1}, u_{2}, u_{3}, u_{4}, u_{5}, u_{6}, w, v\right)$, with $\left|u_{1}\right|=\left|u_{2}\right|=$ $\left|u_{3}\right|=\left|u_{4}\right|=3,\left|u_{5}\right|=5,\left|u_{6}\right|=19,|w|=18,|v|=35$, and $d v=w^{2}+u_{1} u_{2} u_{3} u_{4} u_{5} u_{6}$, all other differentials being zero.

The homotopy Euler characteristic bound yields $\operatorname{rk}_{0}(\mathcal{M})=0$. We will show that $\operatorname{rk}_{0}(\mathcal{N})=0$ by arguing that any extension of the form $(\Lambda a \otimes \mathcal{N}, D)$ cannot have finite-dimensional cohomology. 
Let $U^{\prime}=\left\langle u_{1}, \ldots, u_{5}\right\rangle$ and denote $\Lambda^{\left\{i_{1}, \ldots, i_{k}\right\}} U^{\prime}:=\oplus_{m=1}^{k} \Lambda^{i_{m}} U^{\prime}$. For degree reasons, any such $D$ satisfies $D u_{i}=\alpha_{i} a^{2}$, for $i=1, \ldots, 4, D u_{5}=\alpha_{5} a^{3}, D u_{6}=\alpha_{6} a^{10}+\mu a w+\Phi$, and

$$
D v=w^{2}+u_{1} u_{2} u_{3} u_{4} u_{5} u_{6}+w \Psi+u_{6} \Gamma+\Omega,
$$

where the $\alpha_{i}$ and $\mu$ are scalars, $\Phi, D w \in \Lambda^{+} a \otimes \Lambda^{+} U^{\prime}, \Gamma \in \Lambda^{\geqslant 3} a \otimes \Lambda^{\{1,3\}} U^{\prime}$ and $\Psi, \Omega \in \Lambda^{+} a \otimes \Lambda U^{\prime}$.

Our strategy is to show that each $\alpha_{i}=0$, and then to follow a similar argument as in previous examples. Applying $D$ to (10) and equating terms that contain $u_{6}$, we find

$$
0=\sum_{i=1}^{4} \pm \alpha_{i} a^{2} u_{1} \cdots \hat{u}_{i} \cdots u_{4} u_{5}+u_{1} \cdots u_{4} \alpha_{5} a^{3}-D \Gamma .
$$

Since $D \Gamma \in \Lambda^{\geqslant 5} a \otimes \Lambda U^{\prime}$, we immediately find $\alpha_{i}=0$ for $i=0, \ldots, 5$, and so $D U^{\prime}=0$. Now, $0=D^{2} u_{6}=\mu a D w$. So either $\mu=0$ or $D w=0$. Moreover, the $w$ component of $D^{2} v$ being zero implies that

$$
0=2 D w-\mu a u_{1} \cdots u_{5}+\mu a \Gamma .
$$

If $\mu=0$, then this equation implies $D w=0$. On the other hand, suppose $\mu \neq 0$. Since $\Gamma \in \Lambda^{\geqslant 3} a \otimes \Lambda^{\{1,3\}} U$, by multiplying this last equation by $\mu a$, we conclude that in fact $\mu=0$, and hence that $D w=0$.

The equation $D^{2} v=0$ now implies

$$
0=-\alpha_{6} a^{10} u_{1} \cdots u_{5}+\left(\alpha_{6} a^{10}+\Phi\right) \Gamma .
$$

Suppose $\alpha_{6} \neq 0$. Then, upon considering the component of (11) in $\Lambda^{+} a \otimes \Lambda^{1} U^{\prime}$, we find that $\Gamma \in \Lambda^{+} a \otimes \Lambda^{3} U$. Since $\Phi \in \Lambda^{+} a \otimes \Lambda^{\geqslant 2} U^{\prime}$, this shows that $\Phi \Gamma \in \Lambda^{+} a \otimes \Lambda^{5} U^{\prime}$. Thus, (11) now yields $\Gamma=0$ and so $\alpha_{6}=0$ in any case.

The argument so far has shown $\mu=\alpha_{i}=0$ for $i=1, \ldots, 6$. Therefore, for any minimal model $(\Lambda a \otimes \mathcal{N}, D)$, the corresponding pure model satisfies $\left(\Lambda a \otimes \mathcal{N}, D_{\sigma}\right) \cong$ $\left(\Lambda\left(u_{1}, u_{2}, u_{3}, u_{4}, u_{5}, u_{6}\right), 0\right) \otimes\left(\Lambda(a, w, v), D_{\sigma}\right)$. Since $\left(\Lambda(a, w, v), D_{\sigma}\right)$ has homotopy Euler characteristic $\chi_{\pi}=+1$, it cannot have finite-dimensional cohomology, and hence neither can $\left(\Lambda a \otimes \mathcal{N}, D_{\sigma}\right)$. As before, it follows that $(\Lambda a \otimes \mathcal{N}, D)$ does not have finite-dimensional cohomology, and hence that $\operatorname{rk}_{0}(\mathcal{N})=0$.

Now consider the product $\mathcal{M} \otimes \mathcal{N}$. We show that $\operatorname{rk}_{0}(\mathcal{M} \otimes \mathcal{N}) \geqslant 1$, by displaying an extension $(\Lambda a \otimes \mathcal{M} \otimes \mathcal{N}, D)$, in which $H(\Lambda a \otimes \mathcal{M} \otimes \mathcal{N}, D)$ is finite-dimensional. The differential is as follows: $D x=u_{1} u_{2} u_{3} a^{2}, D y=x^{2}+2 u_{1} u_{6} a, D u_{1}=D u_{2}=D u_{3}=$ $D u_{4}=0, D u_{5}=a^{3}, D u_{6}=u_{2} u_{3} x a, D w=0$ and $D v=w^{2}+u_{1} u_{2} u_{3} u_{4} u_{5} u_{6}+x a u_{4} u_{6}+$ $y a^{2} u_{2} u_{3} u_{4}$. A careful check reveals that this defines a differntial. The associated pure model is now $\left(\Lambda a \otimes \mathcal{M} \otimes \mathcal{N}, D_{\sigma}\right)$ with the only non-trivial differentials $D_{\sigma}(y)=x^{2}$, $D_{\sigma}\left(u_{5}\right)=a^{3}$ and $D_{\sigma}(v)=w^{2}$. This is easily seen to have finite-dimensional cohomology, and hence so does the extension $(\Lambda a \otimes \mathcal{M} \otimes \mathcal{N}, D)$. Therefore, we have $\operatorname{rk}_{0}(\mathcal{M} \otimes$ $\mathcal{N}) \geqslant 1>\operatorname{rk}_{0}(\mathcal{M})+\operatorname{rk}_{0}(\mathcal{N})$.

Discussion 3.4. Recall that $\operatorname{rk}(X)$ denotes the actual toral rank (rather than its rational counterpart) for a finite complex $X$. It is easy to see that the inequality $\operatorname{rk}(X \times Y) \geqslant \operatorname{rk}(X)+\operatorname{rk}(Y)$ holds in general - one takes the obvious product action. The minimal models of Example $3 \cdot 3$ can be realised as the models of honest 'geometrical' spaces. $\mathcal{M}=\Lambda(x, y)$ is the minimal model of $S^{12}$. $=\Lambda\left(u_{1}, u_{2}, u_{3}, u_{4}, u_{5}, u_{6}, w, v\right)$ is the minimal model of a sphere bundle over a 
product of odd spheres. We sketch this: take $S=S^{3} \times S^{3} \times S^{3} \times S^{3} \times S^{5} \times S^{19}$, so that $S$ has minimal model $\Lambda\left(u_{1}, u_{2}, u_{3}, u_{4}, u_{5}, u_{6}\right)$ with zero differential. $S$ has dimension 36 and by pinching the 35-skeleton to a point, we obtain a non-trivial map $q: S \rightarrow S^{36}$. Composing this with a suitable map $p: S^{36} \rightarrow B S O(19)$ to the universal bundle $B S O(19)$, we obtain a non-trivial map $S \rightarrow B S O(19)$. Pulling back the universal real oriented line bundle to one over $S$, via this map, we obtain a real oriented line bundle $\mathbb{R}^{19} \rightarrow E \rightarrow S$. Finally, taking the unit sphere bundle gives a sphere bundle

$$
S^{18} \longrightarrow Y \longrightarrow S \text {. }
$$

We assert that this sphere bundle $Y$ has minimal model $\mathcal{N}$, as in Example $3 \cdot 3$. Our assertion can be justified by a consideration of the possible forms that the minimal model of $Y$ can take, bearing in mind the construction of $Y$. Our main point here is simply to indicate how $\mathcal{N}$ corresponds to the minimal model of a reasonable space. As in Example $3 \cdot 3, \operatorname{rk}_{0}(X)=\operatorname{rk}_{0}(Y)=0$ and this is sufficient to conclude $\operatorname{rk}(X)=\operatorname{rk}(Y)=0$. On the other hand, we have $\operatorname{rk}_{0}(X \times Y) \geqslant 1$, but this is not sufficient to conclude $\operatorname{rk}(X \times Y) \geqslant 1$. We are left with the following intriguing question: does this space $X \times Y$ admit an almost-free circle action? Note that by [Halb, proposition $4 \cdot 2$ ], there is a simply-connected, finite complex that admits a free circle action and which has the rational homotopy type of $X \times Y$.

The preceding examples and discussion give rise to a number of interesting questions. Generally, it would be useful to have conditions under which the equality $\operatorname{rk}_{0}(X \times Y)=\mathrm{rk}_{0}(X)+\mathrm{rk}_{0}(Y)$ either holds, or does not hold. Various special cases are also of interest. For instance, we can ask when does $\operatorname{rk}_{0}\left(X \times S^{2 n+1}\right)=\operatorname{rk}_{0}(X)+1$ ? We note that Halperin has an example in which $\operatorname{rk}_{0}\left(X \times S^{2 n+1}\right)>\mathrm{rk}_{0}(X)+1$. As another special case we could ask whether, for an $n$-fold product of a space with itself, we have $\operatorname{rk}_{0}\left(X^{n}\right)=n \mathrm{rk}_{0}(X)$ ? At present, we know of no example where equality does not hold. Finally, we note that, as in the discussion above, it is reasonable to ask all these questions in the integral setting, too.

\section{3·3. The Gottlieb group}

An interesting suggestion arising from Theorem $2 \cdot 3$ is a connection between $\operatorname{rk}_{0}(X)$ and the dimension of the Gottlieb group. We recall that the $n$th Gottlieb group is the subgroup of $\pi_{n}(X)$ of elements $\alpha=[f]$ such that $(f$, id $): S^{n} \vee X \rightarrow X$ extends to a continuous map $\mathbf{S}^{n} \times X \rightarrow X$. In terms of a minimal model $(\Lambda W, d)$, this corresponds [FHT, p. 392] to the subspace

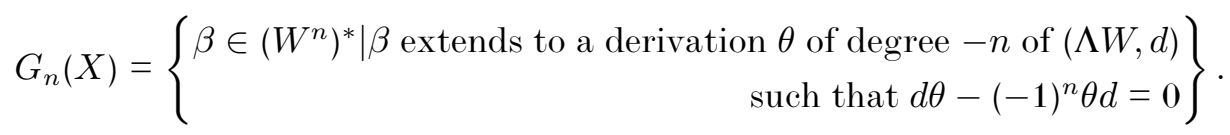

In the two-stage case, with all generators of odd degree, the minimal model can be written so that $\operatorname{dim} V=\operatorname{dim} G_{*}(X)$. Thus we have the following observation:

Corollary 3·5. Suppose $X$ has minimal model that is two-stage with odd-degree generators only. Suppose that $G_{*}(X)$ denotes the rational Gottlieb group of $X$. Then

$$
\operatorname{dim} H(X) \geqslant 2^{\operatorname{dim} G_{*}(X)} .
$$

An examination of Example $3 \cdot 2$ shows that we can have $\operatorname{rk}_{0}(X)>\operatorname{dim} G_{*}(X)$. Next, we illustrate that the dimension of the Gottlieb group can exceed the toral rank by an arbitrary amount. 
Example 3.6. For each $n \geqslant 1$, we describe an $(n+1)$-stage model $\mathcal{M}_{n}$. Set $\mathcal{M}_{n}=$ $\Lambda\left(x_{1}, x_{2}, y_{1}, z_{1}, y_{2}, z_{2}, \ldots, y_{n-1}, z_{n-1}, y_{n}\right)$. For degrees we have $\left|x_{i}\right|=3$ and $\left|z_{i}\right|=3$ for each $i$. The degrees of the $y_{i}$ are chosen to be compatible with the differential, which is as follows: $d\left(x_{i}\right)=0$ and $d\left(z_{i}\right)=0$ for each $i$, then

$$
\begin{aligned}
d\left(y_{1}\right) & =x_{1} x_{2} \\
d\left(y_{2}\right) & =x_{1} x_{2} y_{1} z_{1} \\
d\left(y_{3}\right) & =x_{1} x_{2} y_{1} z_{1} y_{2} z_{2} \\
& \vdots \\
d\left(y_{n}\right) & =x_{1} x_{2} y_{1} z_{1} y_{2} z_{2} \cdots y_{n-1} z_{n-1} .
\end{aligned}
$$

For each $n$, one can show that $\operatorname{dim} G_{*}(X)=n$, but $\operatorname{rk}_{0}(X)=1$. Further, a direct computation shows that $\operatorname{dim} H(X)=(1 / 3) 4^{n+1}+2 / 3$.

These considerations suggest the following question:

Question 3.7. Let $X$ be a finite complex with rational homotopy groups non-zero in odd degrees only. When is $\operatorname{dim} H(X) \geqslant 2^{\operatorname{dim} G_{*}(X)}$ ?

In spite of the apparent difficulty in establishing Conjecture $1 \cdot 1$, it would appear that the conjectured lower bound of $2^{\mathrm{rk}_{0}(X)}$ underestimates the dimension of the cohomology, in many cases quite seriously. This is supported by examples such as Example 3·6, [APb, exercise 4.5] and analogous computations of the cohomology of nilpotent Lie algebras, such as $[\mathbf{A C J}]$. It is possible that the lower bound suggested in Question 3.7 might give a closer estimate in some cases.

Acknowledgements. It is our pleasure to thank Yves Félix and Steve Halperin for their input. They have each generously shared with us their expertise in concocting examples of the sort germane to this paper. In particular, Example 3.1 is due to Yves Félix, and Example $3 \cdot 3$ and the discussion that follows it are entirely due to Steve Halperin.

\section{REFERENCES}

[ACJ $]$ G. Armstrong, G. Cairns and B. Jessup. Explicit Betti numbers for a family of nilpotent lie algebras. Proc. Amer. Math. Soc. 125 (1997), 381-385.

[AH] C. Alldiy and S. Halperin. Lie group actions on spaces of finite rank. Q. J. Math. 28 (1978), 63-76.

[APa C. Allday and V. Puppe. Bounds on the torus rank. Transformation Groups, Poznan 1985, Lecture Notes in Math., vol. 1217 (Springer, 1985), pp. 1-10.

$[\mathrm{APb}]$ C. Allday and V. Puppe. On the localization theorem at the cochain level and free torus actions. Algebraic Topology, Göttingen 1984 (Springer, 1985), pp. 1-16.

[DS] C. Deninger and W. Singhof. On the cohomology of nilpotent lie algebras. Bull. Soc. Math. France 116 (1988), 3-14.

[FHT] Y. Félix, S. Halperin and J.-C. Thomas. Rational homotopy theory. Graduate Texts in Mathematics, vol. 205 (Springer-Verlag, 2001).

[Hala] S. Halperin. Finiteness in the minimal models of Sullivan. Trans. Amer. Math. Soc. 230 (1977), 173-199.

[Halb] S. Halperin. Rational homotopy and torus actions. Aspects of Topology, London Math. Soc. Lecture Notes, vol. 93. (Cambridge Univ. Press, 1985), pp. 293-306.

[Jes] B. Jessup. L-S category and homogeneous spaces. J. Pure Appl. Algebra 65 (1990), 45-56.

[Whi] G. W. Whitenead. Elements of homotopy theory. Graduate Texts in Mathematics, vol. 61, (Springer-Verlag, 1978). 